\title{
BOUNDED HOLOMORPHIC FUNCTION WITH SOME BOUNDARY BEHAVIOR IN THE UNIT BALL OF $C^{n}$
}

\author{
Toshio Matsushima
}

\section{Introduction}

As is well known, the following theorem holds ([3]).

FATOU's THEOREM. If $f(z)$ is a bounded holomorphic function in the unit disk of $\boldsymbol{C}$, then the limit

$$
\lim _{r \rightarrow 1} f(r \zeta)
$$

exists for almost every point $\zeta$ on the unit circle of $\boldsymbol{C}$.

A similar theorem for bounded holomorphic functions in the unit ball of $\boldsymbol{C}^{n}$ for $n \geq 2$ also has been proved in [6]. These theorems show a kind of mildness of bounded holomorphic functions, which was one of the back-grounds of the inner function conjecture (for the details, see [6]). Later the existence of an inner function was proved by Aleksandrov [1] and Löw [4], which causes interest in the bounded holomorphic functions with wild boundary behavior along a radius of the ball.

As a study of boundary behavior along a radius of functions $f$ defined in the unit disk or ball, we consider the following set:

$$
\bigcap_{T<1} \overline{\{f(t \zeta): T<t<1\}}
$$

where $\zeta$ is a boundary point. This set is called the radial cluster set of $f(z)$ at $\zeta$. When the limit of $f(z)$ along the radius terminating at $\zeta$ exists, this set consists of one point. In [5], the author has shown that various sets appear as the radial cluster sets of holomorphic functions.

In this paper we deal with the following problem:

Does there exist a bounded holomorphic function in the unit ball of arbitrary dimension whose radial cluster set is "big" at every point belonging to a given subset of the boundary of the unit ball?

Our main theorem states the following as an answer to this problem:

Received September 11, 2000; revised December 11, 2000. 
MaIN THEOREM. Let $\left\{\zeta_{k}\right\}_{k=1}^{m}$ be an arbitrary discrete subset of the boundary of the unit ball of $\boldsymbol{C}^{n}$, where $1 \leq m \leq+\infty, n \geq 1$ and $\zeta_{k} \neq \zeta_{l}$ if $k \neq l$. Then there exists a bounded holomorphic function $f(z)$ in the unit ball of $\boldsymbol{C}^{n}$ whose radial cluster set at $\zeta_{k}$

$$
\bigcap_{T<1} \overline{\left\{f\left(t \zeta_{k}\right): T<t<1\right\}}
$$

contains a closed disk of positive radius for all $k$.

This gives also some type of counter part of Fatou's theorem of arbitrary dimension. We prove Main theorem in Section 3.

\section{Construction of the basic function}

Lemma 1. Let $(2 \pi \boldsymbol{T})^{n}=\boldsymbol{R}^{n} /(2 \pi \boldsymbol{Z})^{n}$ be a torus of dimension $n$, and let $\left[x_{1}, x_{2}, \ldots, x_{n}\right] \in(2 \pi \boldsymbol{T})^{n}$ denote the residue class of modulus $(2 \pi \boldsymbol{Z})^{n}$ to which $\left(x_{1}, x_{2}, \ldots, x_{n}\right)$ belongs. For $\left(\omega_{1}, \omega_{2}, \ldots, \omega_{n}\right) \in \boldsymbol{R}^{n}$, define the map

$$
\varphi:[0,+\infty) \ni t \mapsto\left[x_{1}+2 \pi \omega_{1} t, x_{2}+2 \pi \omega_{2} t, \ldots, x_{n}+2 \pi \omega_{n} t\right] \in(2 \pi \boldsymbol{T})^{n}
$$

for arbitrarily fixed $\left(x_{1}, x_{2}, \ldots, x_{n}\right) \in \boldsymbol{R}^{n}$. Then the image of $\varphi$ is dense in $(2 \pi \boldsymbol{T})^{n}$ if and only if $\omega_{1}, \omega_{2}, \ldots, \omega_{n}$ are linearly independent over $\boldsymbol{Z}$.

This map is classically known as Kronecker's flow. See, for example, [2] for Lemma 1.

Lemma 2. Let $K$ and $L$ be positive real numbers which are linearly independent over $\boldsymbol{Z}$. Define

$$
F(z)=\exp (2 \pi i K z)+\exp (2 \pi i L z) \text { for } z \in \boldsymbol{C} .
$$

Then $\{F(t): t \geq 0\}$ is a dense subset of $\{w \in C:|w| \leq 2\}$ and

$$
|F(z)| \leq \exp (-2 \pi K \operatorname{Im} z)+\exp (-2 \pi L \operatorname{Im} z) \quad \text { for } z \in \boldsymbol{C} .
$$

Proof. We first consider $F(z)$ for $z=t$ in $[0,+\infty)$ :

$$
F(t)=\exp (2 \pi i K t)+\exp (2 \pi i L t) .
$$

For all $w \in\{w \in \boldsymbol{C}:|w| \leq 2\}$, there exist $w_{1}, w_{2} \in \boldsymbol{C}$ such that $\left|w_{1}\right|=\left|w_{2}\right|=1$ and $w=w_{1}+w_{2}$. Since the image of the map $t \mapsto[2 \pi K t, 2 \pi L t] \in(2 \pi \boldsymbol{T})^{2}$ is dense in $(2 \pi \boldsymbol{T})^{2}$ by Lemma 1 , we can find $(\exp (2 \pi i K t), \exp (2 \pi i L t))$ which is arbitrarily near to $\left(w_{1}, w_{2}\right)$ in $\boldsymbol{C}^{2}$. This proves the first statement.

By the triangle inequality,

$$
\begin{aligned}
|F(z)| & \leq|\exp (2 \pi i K(\operatorname{Re} z+i \operatorname{Im} z))|+|\exp (2 \pi i L(\operatorname{Re} z+i \operatorname{Im} z))| \\
& =\exp (-2 \pi K \operatorname{Im} z)+\exp (-2 \pi L \operatorname{Im} z) .
\end{aligned}
$$

Q.E.D. 
Throughout this paper, we use the following notation:

$$
\begin{gathered}
\langle z, w\rangle=\sum_{k=1}^{n} z_{k} \overline{w_{k}}, \quad|z|=\sqrt{\langle z, z\rangle} \text { for } z=\left(z_{1}, \ldots, z_{n}\right), w=\left(w_{1}, \ldots, w_{n}\right) \in \boldsymbol{C}^{n} \\
B_{n}=\left\{z \in \boldsymbol{C}^{n}:|z|<1\right\}, \quad \partial B_{n}=\left\{z \in \boldsymbol{C}^{n}:|z|=1\right\} \quad \text { and } \quad \Delta=B_{1} .
\end{gathered}
$$

Lemma 3. Let $K$ and $L$ be as in Lemma 2. For an arbitrary point $\zeta \in \partial B_{n}$, define

$$
\tilde{f}(z)=\exp (-2 \pi i K \log (1-\langle z, \zeta\rangle))+\exp (-2 \pi i L \log (1-\langle z, \zeta\rangle)), \quad z \in B_{n},
$$

where the argument $\theta$ of the logarithm is taken as $-\pi<\theta \leq \pi$. Then $\tilde{f}(z)$ is holomorphic in $B_{n}$ and $\|\tilde{f}\|=\sup _{z \in B_{n}}|f(z)| \leq \exp \left(\pi^{2} K\right)+\exp \left(\pi^{2} L\right)$. Moreover, the image of the radius of $B_{n}$ terminating at $\zeta$ by $\tilde{f}(z)$ is a dense subset of $\{w \in C:|w| \leq 2\}$.

Proof. Since $|\langle z, \zeta\rangle|<1$ for all $z$ in $B_{n}$ by Schwarz inequality, the function $\langle\cdot, \zeta\rangle$ maps $B_{n}$ onto $\Delta$, and hence, the image of $B_{n}$ by $1-\langle\cdot, \zeta\rangle$ is $\{z \in C:|z-1|<1\}$. Since the argument is chosen as in Lemma 3, then we have

$$
-\frac{\pi}{2}<\arg (1-\langle z, \zeta\rangle)<\frac{\pi}{2}
$$

and $-\log (1-\langle z, \zeta\rangle)$ is a single valued holomorphic function in $z$, which shows that $\tilde{f}(z)$ is holomorphic in $B_{n}$. On the other hand, we easily have

$$
\begin{aligned}
|\tilde{f}(z)| & \leq \exp (2 \pi K \arg (1-\langle z, \zeta\rangle))+\exp (2 \pi L \arg (1-\langle z, \zeta\rangle)) \\
& <\exp \left(\pi^{2} K\right)+\exp \left(\pi^{2} L\right)
\end{aligned}
$$

by Lemma 2 and the fact that $\arg (1-\langle z, \zeta\rangle)<\pi / 2$. Note that the radius of $B_{n}$ terminating at $\zeta$ is the set $\{t \zeta: 0 \leq t<1\}$. Therefore, by Lemma 2 and

$$
\tilde{f}(t \zeta)=\exp (-2 \pi i K \log (1-t))+\exp (-2 \pi i L \log (1-t)),
$$

we conclude that the image of the radius terminating at $\zeta$ is a dense subset of $\{w \in C:|w| \leq 2\}$.

Q.E.D.

Remarks. (1) For an arbitrary positive real number $M$, let

$$
h(z)=\frac{M}{\exp \left(\pi^{2} K\right)+\exp \left(\pi^{2} L\right)} \tilde{f}(z) .
$$

Suppose that $K$ and $L$ are positive real numbers which are linearly independent over $\boldsymbol{Z}$. Then, by Lemma 3, $\|h\| \leq M$ and the image of the radius of $B_{n}$ terminating at $\zeta$ is a dense subset of the closed disk

$$
\left\{w \in C:|w| \leq \frac{2 M}{\exp \left(\pi^{2} K\right)+\exp \left(\pi^{2} L\right)}\right\} .
$$


(2) The function $h(z)$ has the only singularity $\zeta$ as a function on the closure $\overline{B_{n}}$ of $B_{n}$. Clearly, $h(z)$ is a continuous function in $\overline{B_{n}} \backslash\{\zeta\}$ and $|h(z)| \leq M$ for all $z \in \overline{B_{n}} \backslash\{\zeta\}$, since $-\pi / 2<\arg (1-\langle z, \zeta\rangle)<\pi / 2$ for all $z \in \overline{B_{n}} \backslash\{\zeta\}$.

(3) The radial cluster set of $h(z)$ at $\zeta$ is a closed disk as in (1), namely

$$
\bigcap_{T<1} \overline{\{h(t \zeta): T<t<1\}}=\left\{w \in C:|w| \leq \frac{2 M}{\exp \left(\pi^{2} K\right)+\exp \left(\pi^{2} L\right)}\right\} .
$$

\section{Proof of the main theorem}

We first prove the following lemma on the radial cluster set:

Lemma 4. Let $g_{1}(z)$ and $g_{2}(z)$ be functions which are defined in $B_{n}$ and let $\Lambda$ be the radial cluster set of $g_{1}(z)$ at a point $\zeta \in \partial B_{n}$. Suppose that $g_{2}(z)$ has the radial limit $\alpha$ at the point $\zeta$, i.e.,

$$
\lim _{t \rightarrow 1} g_{2}(t \zeta)=\alpha
$$

Then the radial cluster set of $g_{1}+g_{2}$ at $\zeta$ is $\Lambda+\alpha$.

Proof. Let $\varepsilon$ be an arbitrary positive real number and $p$ an arbitrary point in $\Lambda$. By assumptions of Lemma 4 , there exist a sequence $\left\{t_{k}\right\}_{k=1}^{\infty}$ with $t_{k} \uparrow 1$ as $k \rightarrow \infty$ and a natural number $N$ such that if $k>N$, then

$$
\left|g_{1}\left(t_{k} \zeta\right)-p\right|<\frac{\varepsilon}{2} \quad \text { and } \quad\left|g_{2}\left(t_{k} \zeta\right)-\alpha\right|<\frac{\varepsilon}{2} .
$$

Hence if $k>N$, then

$$
\left|(p+\alpha)-\left(g_{1}+g_{2}\right)\left(t_{k} \zeta\right)\right| \leq\left|p-g_{1}\left(t_{k} \zeta\right)\right|+\left|\alpha-g_{2}\left(t_{k} \zeta\right)\right|<\varepsilon,
$$

which yields that $p+\alpha$ is a point of the radial cluster set of $g_{1}+g_{2}$ at $\zeta$. Consequently, we see

$$
\Lambda+\alpha \subset \bigcap_{T<1} \overline{\left\{\left(g_{1}+g_{2}\right)(t \zeta): T<t<1\right\}} .
$$

Conversely, suppose that $s$ is a point of the radial cluster set of $g_{1}+g_{2}$ at $\zeta$, which is not in $\Lambda+\alpha$. Then $r:=s-\alpha \notin \Lambda$, and so we can find some positive number $d$ such that $\{w:|w-r| \leq 2 d\} \cap \Lambda=\phi$. Take an arbitrary monotone increasing sequence $\left\{q_{k}\right\}_{k=1}^{\infty}$ of non-negative numbers which converges to 1 . Then, there exist a natural number $N_{d}$ such that $\left|g_{1}\left(q_{k} \zeta\right)-r\right| \geq 2 d$ if $k>N_{d}$. Thus if $k>N_{d}$, then

$$
\begin{aligned}
\left|\left(g_{1}+g_{2}\right)\left(q_{k} \zeta\right)-s\right| & =\left|g_{1}\left(q_{k} \zeta\right)+g_{2}\left(q_{k} \zeta\right)-(r+\alpha)\right| \\
& =\left|\left(g_{1}\left(q_{k} \zeta\right)-r\right)+\left(g_{2}\left(q_{k} \zeta\right)-\alpha\right)\right| \\
& \geq|| g_{1}\left(q_{k} \zeta\right)-r|-| g_{2}\left(q_{k} \zeta\right)-\alpha|| .
\end{aligned}
$$


Clearly $\left|g_{2}\left(q_{k} \zeta\right)-\alpha\right|<\varepsilon$ for an arbitrary positive number $\varepsilon$ if $k$ is suffeciently large. If we choose $\varepsilon$ less than $d$,

$$
\left|\left(g_{1}+g_{2}\right)\left(q_{k} \zeta\right)-s\right| \geq 2 d-\varepsilon>d,
$$

which contradicts the fact that $s$ is a point of the radial cluster set of $g_{1}+g_{2}$ at $\zeta$. Thus we see

$$
\bigcap_{T<1} \overline{\left\{\left(g_{1}+g_{2}\right)(t \zeta): T<t<1\right\}} \subset \Lambda+\alpha .
$$

Therefore we conclude that

$$
\bigcap_{T<1} \overline{\left\{\left(g_{1}+g_{2}\right)(t \zeta): T<t<1\right\}}=\Lambda+\alpha .
$$

Proof of the main theorem. Let $M$ be an arbitrary positive number, and let

$$
\begin{aligned}
f_{k}(z)= & \frac{M}{\exp \left(\pi^{2} K\right)+\exp \left(\pi^{2} L\right)}\left(\exp \left(-2 \pi i K \log \left(1-\left\langle z, \zeta_{k}\right\rangle\right)\right)\right. \\
& \left.+\exp \left(-2 \pi i L \log \left(1-\left\langle z, \zeta_{k}\right\rangle\right)\right)\right)
\end{aligned}
$$

for $z$ in $B_{n}$, where $K$ and $L$ are positive real numbers which are linearly independent over $\boldsymbol{Z}$. We choose the branch of the logarithm as in Lemma 3. Note that each $f_{k}(z)$ is the same function as $h(z)$ in Remarks if we substitute $\zeta_{k}$ for $\zeta$. Every $f_{k}(z)$ is holomorphic in $B_{n}$.

When $m$ is finite, let

$$
f(z)=\frac{1}{m} \sum_{k=1}^{m} f_{k}(z)
$$

Then $f(z)$ is holomorphic in $B_{n}$ and $\|f\| \leq M$ by (1) in Remarks. We write

$$
f(z)=\frac{1}{m} f_{1}(z)+\frac{1}{m} \sum_{k=2}^{m} f_{k}(z)
$$

The radial cluster set of the first term at $\zeta_{1}$ is the closed disk

$$
\left\{w \in C:|w| \leq \frac{1}{m} \cdot \frac{2 M}{\exp \left(\pi^{2} K\right)+\exp \left(\pi^{2} L\right)}\right\},
$$

and the second term is continuous at $\zeta_{1}$, which are derived by (2) and (3) in Remarks. Hence, by Lemma 4, the radial cluster set of $f(z)$ at $\zeta_{1}$ is the closed disk

$$
\left\{w \in C:\left|w-\frac{1}{m} \sum_{k=2}^{m} f_{k}\left(\zeta_{1}\right)\right| \leq \frac{1}{m} \cdot \frac{2 M}{\exp \left(\pi^{2} K\right)+\exp \left(\pi^{2} L\right)}\right\} .
$$


We can also derive the same result at each $\zeta_{p}$ for $2 \leq p \leq m$, by decomposing $f(z)$ into sum

$$
f(z)=\frac{1}{m} f_{p}(z)+\frac{1}{m} \sum_{k=1, k \neq p}^{m} f_{k}(z) .
$$

These show that the radial cluster set of $f(z)$ at $\zeta_{k}$ is a closed disk for every $k$ with $1 \leq k \leq m$.

When $m$ is infinite, let

$$
f(z)=\sum_{k=1}^{\infty} a_{k} f_{k}(z)
$$

where $\left\{a_{k}\right\}_{k=1}^{\infty}$ is a sequence of positive numbers satisfying $\sum_{k=1}^{\infty} a_{k}=1$. Then

$$
|f(z)| \leq \sum_{k=1}^{\infty}\left|a_{k} f_{k}(z)\right| \leq \sum_{k=1}^{\infty} a_{k}\left\|f_{k}\right\| \leq M \sum_{k=1}^{\infty} a_{k}=M<+\infty,
$$

which shows that $f(z)$ is a bounded holomorphic function in $B_{n}$.

Next, we see the radial cluster set of $f(z)$ at $\zeta_{p}$ for $p \geq 1$. Without loss of generality, we may assume $p=1$. Let

$$
F_{n}(z)=\sum_{k=1}^{n} a_{k} f_{k}(z) \quad \text { for } n=1,2, \ldots
$$

Since $F_{n}(z)$ absolutely and uniformly converges to $f(z)$ on $B_{n}$, there exists a natural number $N_{\varepsilon}$ for any positive $\varepsilon$ such that

$$
\left\|f-F_{n}\right\|<\varepsilon \text { for } n>N_{\varepsilon}
$$

and

$$
\left\|F_{m}-F_{n}\right\|<\varepsilon \text { for } m, n>N_{\varepsilon} .
$$

Note that

$$
\bigcap_{T<1} \overline{\left\{a_{1} f_{1}\left(t \zeta_{1}\right): T<t<1\right\}}=\left\{w \in C:|w| \leq \frac{2 a_{1} M}{\exp \left(\pi^{2} K\right)+\exp \left(\pi^{2} L\right)}\right\} .
$$

Set

$$
\delta=\frac{1}{10} \cdot \frac{2 a_{1} M}{\exp \left(\pi^{2} K\right)+\exp \left(\pi^{2} L\right)} .
$$

By (2), we have a natural number $N$ such that

$$
\left\|F_{n}-F_{N+1}\right\|<\delta \text { for } n>N+1 .
$$

Decompose $F_{N+1}(z)$ as

$$
F_{N+1}(z)=a_{1} f_{1}(z)+\sum_{k=2}^{N+1} a_{k} f_{k}(z) .
$$


Then, the second term is continuous at $\zeta_{1}$, and radial cluster set of $a_{1} f_{1}(z)$ at $\zeta_{1}$ is a closed disk as above. Hence, by Lemma 4, the radial cluster set of $F_{N+1}$ at $\zeta_{1}$ is the closed disk

$$
\bar{D}_{N+1}=\left\{w \in C:\left|w-\sum_{k=2}^{N+1} a_{k} f_{k}\left(\zeta_{1}\right)\right| \leq \frac{2 a_{1} M}{\exp \left(\pi^{2} K\right)+\exp \left(\pi^{2} L\right)}\right\} .
$$

Also, when $n>N+1$, the radial cluster set of $F_{n}(z)$ at $\zeta_{1}$ is the closed disk

$$
\bar{D}_{n}=\left\{w \in C:\left|w-\sum_{k=2}^{n} a_{k} f_{k}\left(\zeta_{1}\right)\right| \leq \frac{2 a_{1} M}{\exp \left(\pi^{2} K\right)+\exp \left(\pi^{2} L\right)}\right\} .
$$

Notice that each $\bar{D}_{n}$ and $\bar{D}_{N+1}$ have the same radius $10 \delta$. We also note that, if $n>N+1$, the distance between the centers of $\bar{D}_{n}$ and $\bar{D}_{N+1}$ is

$$
\left|\sum_{k=2}^{n} a_{k} f_{k}\left(\zeta_{1}\right)-\sum_{k=2}^{N+1} a_{k} f_{k}\left(\zeta_{1}\right)\right|=\left|\sum_{k=N+2}^{n} a_{k} f_{k}\left(\zeta_{1}\right)\right| .
$$

On the other hand, by (3) we have

$$
\left|\sum_{k=N+2}^{n} a_{k} f_{k}\left(t \zeta_{1}\right)\right|=\left|F_{n}\left(t \zeta_{1}\right)-F_{N+1}\left(t \zeta_{1}\right)\right|<\delta
$$

for all $t$ with $0 \leq t<1$. Since $\sum_{k=N+2}^{n} a_{k} f_{k}(z)$ is continuous at $\zeta_{1}$ for every $n>$ $N+1$, letting $t \rightarrow 1$, we obtain that

$$
\left|\sum_{k=N+2}^{n} a_{k} f_{k}\left(\zeta_{1}\right)\right| \leq \delta
$$

Hence the distance between the centers of the disks $\bar{D}_{N+1}$ and $\bar{D}_{n}$ is less than or equel to $\delta$ for $n>N+1$. This indicates that every $\bar{D}_{n}$ contains the closed disk

$$
\bar{D}=\left\{w \in \boldsymbol{C}:\left|w-\sum_{k=2}^{N+1} a_{k} f_{k}\left(\zeta_{1}\right)\right| \leq \delta\right\}
$$

when $n>N+1$. This fact shows that the closure of the image of the radius of $B_{n}$ terminating at $\zeta_{1}$ by $F_{n}(z)$ contains $\bar{D}$.

Take an arbitrary point $\alpha \in \bar{D}$ and an arbitrary positive number $\varepsilon$. By (1), we can choose some natural number $N_{\varepsilon}$ such that

$$
\left|f\left(t \zeta_{1}\right)-F_{n}\left(t \zeta_{1}\right)\right|<\frac{\varepsilon}{2}
$$

for all $t$ with $0 \leq t<1$ and all $n>N_{\varepsilon}$. Set

$$
n=\max \left\{N+1, N_{\varepsilon}\right\}+1 \text {. }
$$


Then, since $\alpha$ belongs to $\bar{D}_{n}$, there exists a sequence $\left\{t_{k}\right\}_{k=1}^{\infty}$ with $t_{k} \uparrow 1$ and a natural number $A_{\varepsilon}$ such that

$$
\left|F_{n}\left(t_{k} \zeta_{1}\right)-\alpha\right|<\frac{\varepsilon}{2} \text { for } k>A_{\varepsilon} .
$$

Thus, for $k>A_{\varepsilon}$, by (4) and (5), we have

$$
\begin{aligned}
\left|f\left(t_{k} \zeta_{1}\right)-\alpha\right| & =\left|f\left(t_{k} \zeta_{1}\right)-F_{n}\left(t_{k} \zeta_{1}\right)+F_{n}\left(t_{k} \zeta_{1}\right)-\alpha\right| \\
& \leq\left|f\left(t_{k} \zeta_{1}\right)-F_{n}\left(t_{k} \zeta_{1}\right)\right|+\left|F_{n}\left(t_{k} \zeta_{1}\right)-\alpha\right|<\varepsilon
\end{aligned}
$$

This shows that $\alpha$ is contained in the radial cluster set of $f(z)$ at $\zeta_{1}$. Hence

$$
\bigcap_{T<1} \overline{\left\{f\left(t \zeta_{1}\right): T<t<1\right\}} \supset \bar{D} .
$$

Q.E.D.

Acknowledgements. The author would like to thank Professor Hirotaka Fujimoto for his encouragemant and smart advice, and also would like to thank Professors Yoshihiro Aihara and Akio Kodama for their useful suggestions.

\section{REFERENCES}

[1] A. B. Aleksandrov, Existence of inner functions in the ball, Mat. Sb., 117 (1982), 147-163.

[2] V. I. Arnold and A. Avez, Problèm Ergodiques de la Mécanique Classique, Gauthier-Villas, 1967.

[ 3 ] P. Fatou, Séries trigonométriques et séries de Taylor, Acta Math., 30 (1906), 335-400.

[4] E. Löw, A construction of inner functions on the unit ball in $\boldsymbol{C}^{p}$, Invent. Math., 67 (1982), 223-229.

[5] T. Matsushima, Image of a radius by a holomorphic function and map on the unit disk of C, Math. J. Toyama Univ., 18 (1995), 97-106.

[6] W. Rudin, Function Theory in The Unit Ball of $\boldsymbol{C}^{n}$, Springer, Berlin, 1980.

Ishikawa National College of Technology

Kita-ChuhJoh, Tsubata

ISHIKAWA, 929-0392

JAPAN

E-mail: matsush@ishikawa-nct.ac.jp 